\title{
Measurement of Cesium-137 and Cobalt-60 Gamma Radiation With a Pressure Ionization Chamber
}

\author{
G. R. Grove ${ }^{1}$
}

\begin{abstract}
The characteristics of the pressure ionization chamber at the National Bureau of Standards have been studied for the gamma radiation from a cesium- 137 source $(0.6614 \mathrm{Mev})$ and from a cobalt-60 source (1.1715 and $1.3316 \mathrm{Mev})$. The dosage rates produced in air by these sources were measured with this chamber with an accuracy of about 2 percent. Dosage rates obtained with an extrapolation chamber and a Victoreen thimble chamber agreed with the values measured by the pressure chamber within the limits of the experimental errors.
\end{abstract}

\section{Introduction}

A study of the charactristics of the pressure ionization chamber at the National Bureau of Standards for X-rays up to $400 \mathrm{kv}$ has been discussed by Taylor, Singer, and Charlton [1] $]^{2}$. The results of their study showed that it was possible to measure accurately the dosage rates of high-voltage X-rays in accordance with the international definition of the roentgen. Taylor and Singer [2] also used this chamber to measure the ionization of the gamma radiation from a radium source and determined a value for the emission constant of radium. At that time it was not feasible to investigate completely the chamber response with quantum energies in the million-volt region. However, Taylor and Singer believed their measurements were accurate to within \pm 0.4 percent.

Radioactive materials of sufficient radioactivity intensity and particularly suited for use with the pressure chamber have become available only recently. The study of the characteristics of the chamber with isotopes having gamma radiation in the energy regions of 0.6 and $1.3 \mathrm{Mev}$ was undertaken to determine the reliability of the chamber for measuring the dosage rates produced by radiation energies up to 1.3 Mev. An extrapolation chamber and a Victoreen thimble chamber were also tested so that their readings could be compared with that of the pressure chamber.

Cesium-137 and cobalt-60 isotopes were obtained from the Oak Ridge National Laboratory for use with the chamber. The cesium isotope emits a gamma ray of $0.6614 \mathrm{Mev}$ [3], and cobalt-60 emits 2 quanta in cascade per disintegration with energies of 1.1715 and $1.3316 \mathrm{Mev}$ [4]. The activities of the cesium and cobalt samples were about 8 and 1.5 curies, respectively.

\section{Apparatus and Procedure}

\subsection{Pressure Chamber}

The diaphragm system and chamber are shown schematically in figure 1 . The source is housed in an aluminum cylindrical spool, which can be moved

1 Present address, Mound Laboratory, Miamisberg, Ohio.

2 Figures in brackets indicate the literature references at the end of this paper

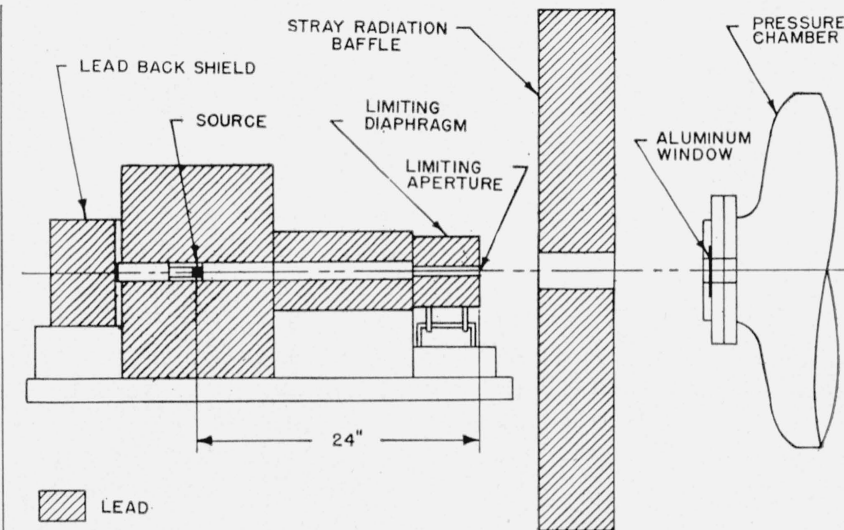

FIGURE 1. Diaphragm system.

axially inside a $1 \frac{1}{2}$-in. diameter aluminum tube to vary the distance between the diaphragm and the source. The diameter of the aluminum tube is twice as large as the diameter of the diaphragm aperture and about three times as large as the diameters of the sources used. The aluminum tubing is supported in containers that are filled with lead shot to reduce the radiation intensity in all directions except along the axis of the tube. A lead wall across the back of the tubing confines the radiation to a forward direction.

The gamma-ray beam defined by the diaphragm passes through an aluminum window $0.8 \mathrm{~mm}$ thick and $5.2 \mathrm{~cm}$ in diameter into the chamber. The cylindrical pressure chamber has an inside length of $7 \mathrm{ft}$ and a diameter of 30 in. Collecting electrodes are arranged in the chamber on a pair of rails in such a way that they can be moved from the outside along the axis of the tank over a range of about 125 $\mathrm{cm}$. The spacing of the electrodes also can be smoothly adjusted from $7.2 \mathrm{~cm}$ up to about $39 \mathrm{~cm}$. These electrodes consist of aluminum plates $75 \mathrm{~cm}$ long and $40 \mathrm{~cm}$ in height. An insulated aluminum collecting plate $25.4 \mathrm{~cm}$ wide and $35 \mathrm{~cm}$ high is located at the center of the grounded electrode. The sections of the grounded electrode around the collecting plate act as guard plates. Equal potential planes are provided in the sensitive ionization volume by means $0.4-\mathrm{mm}$ aluminum guard wires located in planes 
parallel to the electrodes. Each of these wires completely surrounds the volume defined by the plates. These guard wires, which are $2 \mathrm{~cm}$ apart with adjacent wires connected by 2 -megohm metalized resistors, produce a uniformly graded potential between the high-voltage electrode and the grounded guard plates.

Air is pumped into the chamber through a filter and a calcium chloride drier, and in addition, several trays of drier are kept inside the pressure tank to keep the moisture content of the air at a minimum. The pressure of the gas can be read directly from a Bourdon gage with an accuracy of about 0.2 percent, and the temperature of the gas can be determined from a thermometer fastened to the electrode assembly, which can be read through a thick glass window in the rear of the tank with an accuracy of about 0.4 percent.

The potential applied to the high-voltage electrode is supplied by a group of about $500 \mathrm{~B}$ batteries $(45$ $\mathrm{v}$ per battery). Potentials from 500 to $20,000 \mathrm{v}$ are available in steps varying from about 500 to 2,500 v. Using a $100-\mu$ a meter with appropriate multipliers, the plate potential can be measured with an accuracy of about 0.5 percent. A General Electric Co. FP-54 electrometer tube is used in a conventional detector circuit with a galvanometer of approximately $10^{-9} \mathrm{amp} / \mathrm{mm}$ sensitivity. A Rubicon potentiometer connected to the collecting plate through a S. S. White resistor supplies the necessary current through the resistor to buck out the current from the collecting plate and maintain it at ground potential. With the grid of the electrometer tube also connected to the collecting electrode, the compensation current can be adjusted to exactly neutralize the ionization current as indicated by a balance of the galvanometer.

The dosage rate supplied to the ionization chamber under saturation conditions can then be determined from a knowledge of the effective volume of air defined by the collecting plate and the diaphragm aperture along with the values of the grid resistor and the potentiometer voltage necessary to balance the detector.

If the response of the pressure chamber with plate voltage and plate separation at various pressures is internally consistent and is in agreement with the expected characteristic response of a parallel plate chamber, the values measured can be relied upon as representing the true dosage rates.

Using the cesium-137 source and a constant plate separation of $35.14 \mathrm{~cm}$, the ionization current was measured as a function of the plate voltage for several different pressures ranging from 4.81 to $8.90 \mathrm{~atm}$ corrected to standard temperature and pressure (see fig. 2). The values of the ionization currents per unit volume of standard air obtained by the extrapolation of the saturation curves to an infinite electric field by the technique of Jaffe and Zanstra [5] and making the corrections due to air absorption, agreed within 1 percent with their average value. With a constant pressure of $8.93 \mathrm{~atm}$, the ionization current response to plate voltage was measured for

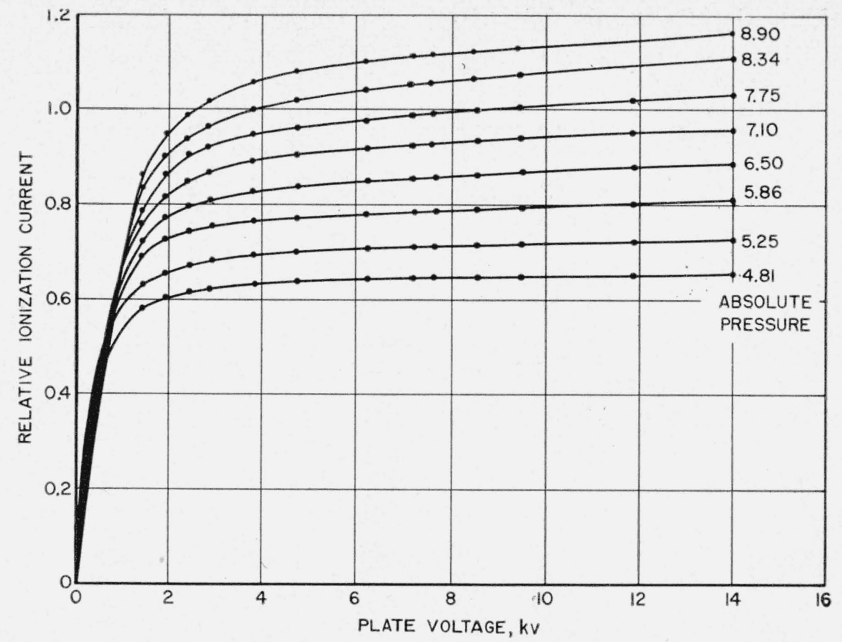

FIGURE 2. Voltage saturation curves for different chamber pressures.

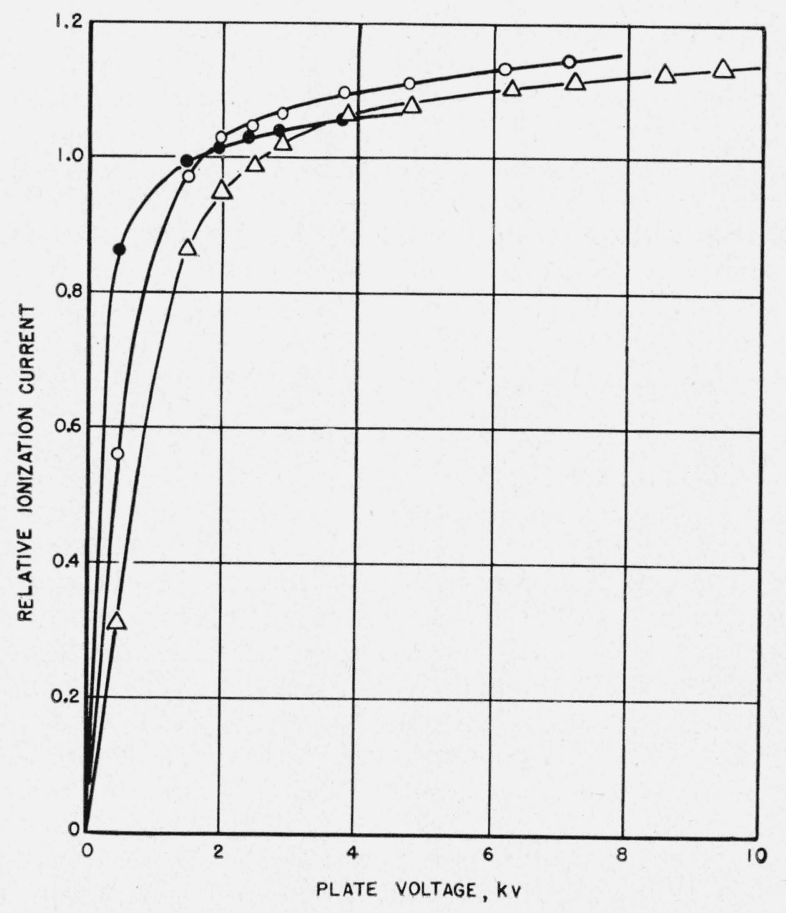

Figure 3. Voltage saturation curves for different plate separations.

, $9.98 \mathrm{~cm} ; \bigcirc, 23.96 \mathrm{~cm} ; \triangle, 35.14 \mathrm{~cm}$.

different plate separations ranging from 7.18 to 37.94 $\mathrm{cm}$. The saturation curves for three of the plate separations are shown in figures 3 and 4 . With the smaller plate separations, the ionization current increases more rapidly in the lower plate voltage range than does the current from a larger plate separation, due to the greater field strength. When the plate separation is not large enough, as is the case of 9.98 $\mathrm{cm}$ at $8.93 \mathrm{~atm}$ (fig. 3 ), some electrons are collected by the electrodes before they expend all of their recoil energies by ionization. From the complete family of saturation curves at a constant pressure, a curve 


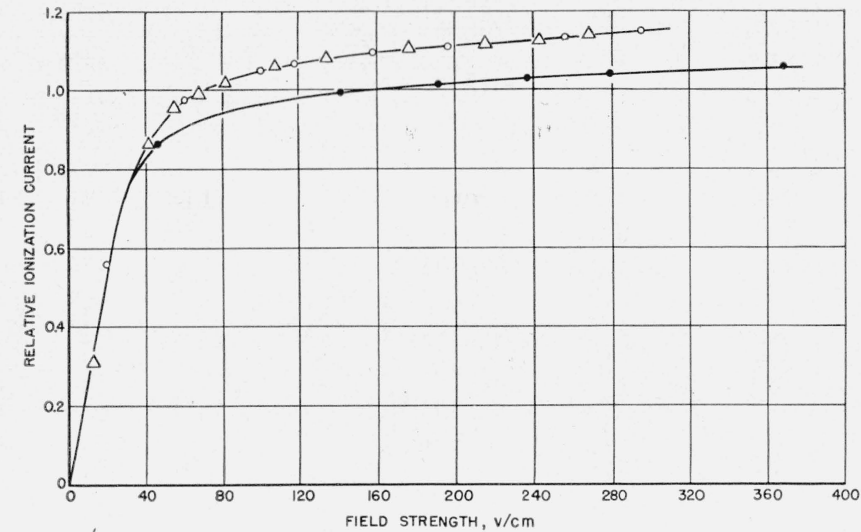

FIGURE 4. Field saturation curves for different plate separations.

- $9.98 \mathrm{~cm} ; \bigcirc, 23.96 \mathrm{~cm} ; \triangle, 35.14 \mathrm{~cm}$.

of the ionization current versus the plate separation with constant electric field was determined. The ionization current was found to fall off for plate separations less than about $150 \mathrm{~cm}$ of equivalent standard air. When corrected for the diameter of the gamma-ray beam and the chamber pressure, a minimum sufficient plate separation of about $120 \mathrm{~cm}$ for $0.661-\mathrm{Mev}$ radiation was found.

A similar procedure was followed in measuring the radiation intensity from the cobalt-60 source. For these quantum energies a minimum plate separation of about $400 \mathrm{~cm}$ of standard air was found necessary to obtain the maximum collecting rate of the ions with a constant electric field. This

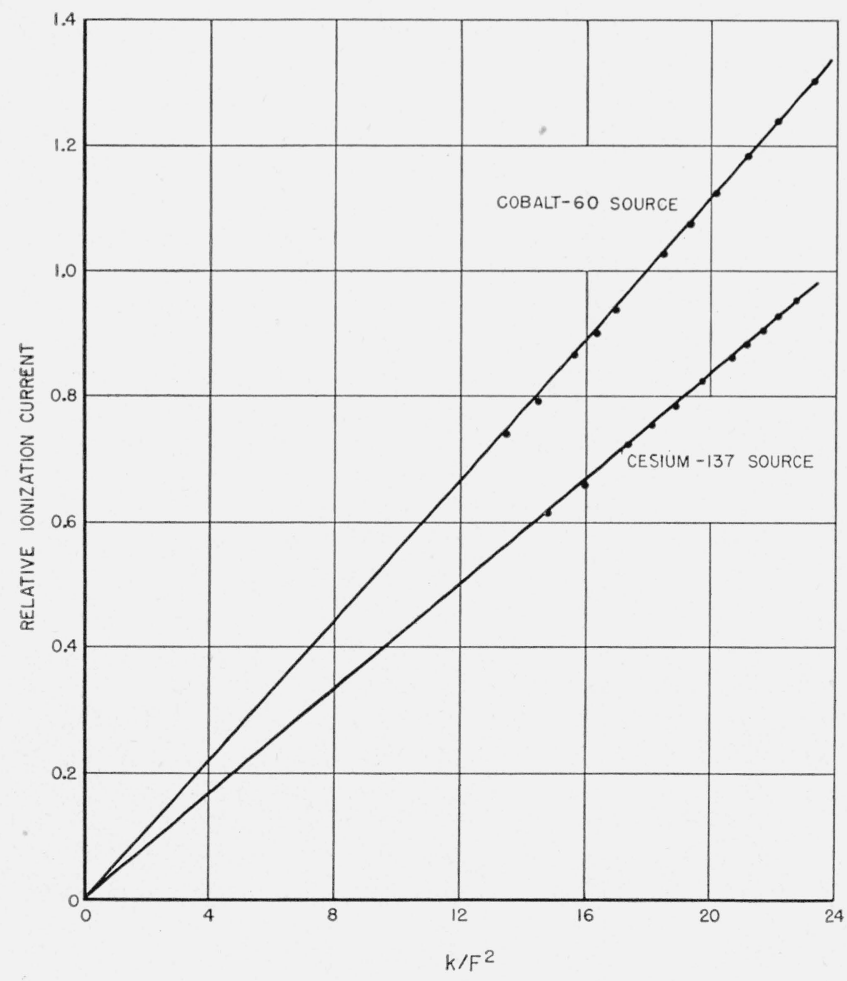

FiguRE 5. Inverse-square plot for the source-diaphragm distance $\left(F^{\prime}\right)$. corresponds to a plate separation of about $335 \mathrm{~cm}$ of equivalent standard air when corrected for the beam diameter.

The dosage rates at the limiting diaphragm surface for the particular cesium and cobalt sources measured in the manner just described were $6.91 \pm 0.07$ and $5.10 \pm 0.05 \mathrm{r} / \mathrm{hr}$, respectively. These values need to be corrected only for the air and aluminum absorption and for the diaphragm leakage.

A test of the inverse-square response was made with the pressure chamber for the two sources. The distance, $F$, between the center of the source and the limiting diaphragm surface was varied, and the concomitant ionization current was measured. These data are shown in figure 5, from which it is seen that $F$ is very nearly equal to the effective source-diaphragm distance.

\subsection{Extrapolation Chamber}

A parallel-plate extrapolation chamber [6] also was used to measure the dosage rates of the two sources. This chamber consists of two Lucite disks, the inner surfaces of which are painted with a conducting aquadag dispersion. Around each disk there is a concentric guard ring (fig. 6). The Lucite disks are supported in, but insulated from, brass rings, one of which is threaded into the other so that the plate separation can be varied. A complete revo-

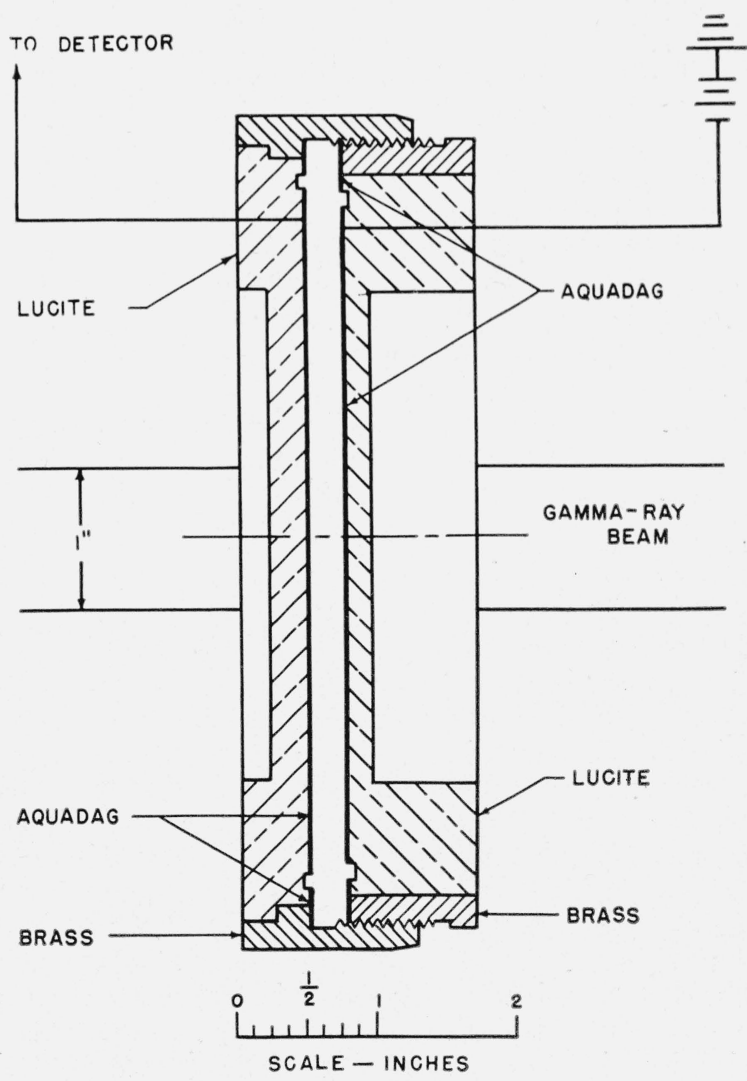

FIgure 6. Sectional view of extrapolation chamber showing approximate size of gamma-ray beam. 


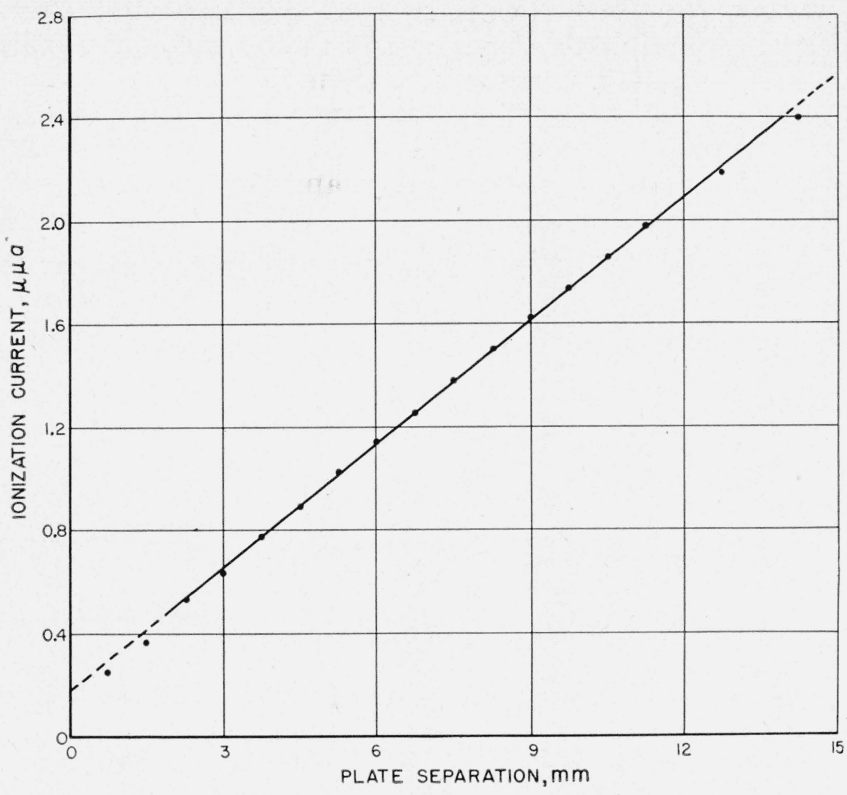

Figure 7. Extrapolation chamber curve for the cesium-13? source.

lution of one of the plates changes the plate separation by $0.150 \mathrm{~cm}$, which is about 8 percent of the maximum plate separation.

By the use of this chamber with a shielded condenser-potentiometer circuit and a vibrating-reed electrometer as an indicator, the ionization current was measured as a function of the plate separation. A curve for the cesium source is shown in figure 7 . From the slope of the straight line determined by the most points, the average ionization current per centimeter can be found. This quantity, together with the values of the temperature, pressure, and the area of the diaphragm aperture, determines a dosage rate at the limiting diaphragm surface. For the cesium and cobalt sources, dosage rates of 6.72 \pm 0.13 and $5.20 \pm 0.10 \mathrm{r} / \mathrm{hr}$, respectively, were calculated. These values need to be corrected only for air and Lucite absorption and for the diaphragm leakage.

\subsection{Thimble Chamber}

A 25- $r$ Victoreen thimble chamber was also used to measure the dosage rates of the two isotopes. This chamber and charger unit had been previously calibrated for radium radiation, and a scale correction was determined with an accuracy of about 2 percent. With this chamber the dosage rates were measured for the two sources at a sufficient distance from the diaphragm, such that the largest dimension of the sensitive volume of the chamber was less than one-half the diameter of the umbra portion of the radiation. With the same geometry, readings were taken with different thicknesses of Lucite shells around the sensitive volume. The rate of ionization in the chamber at first increased with shell thickness due to the approach to electronic equilibrium, and then decreased with additional shell thickness due to absorption in the Lucite. By extrapolating the slope of the line due to absorption to zero shell thickness, a dosage rate was determined. This value is taken to be a good approximation to the ionization rate with electronic equilibrium and no Lucite absorption. The shell thicknesses necessary for electronic equilibrium were estimated to be about $1.0 \mathrm{~mm}$ for $0.661 \mathrm{-Mev}$ radiation and $1.3 \mathrm{~mm}$ for $1.3 \mathrm{-Mev}$ radiation. The extrapolated values were about 8.8 percent for cesium and 4.5 percent for cobalt higher than the respective values with electronic equilibrium shell thicknesses. Extrapolated values of $7.04 \pm 0.21$ and $5.20 \pm 0.15 \mathrm{r} / \mathrm{hr}$ were calculated for the cesium and cobalt sources, respectively. These values are corrected for temperature and pressure and are for the same source-limiting aperture distance used with the other two chambers. These values can then be compared directly with the pressure-chamber results.

\section{Corrections and Results}

The sensitive volume of the pressure ionization chamber is determined by the length of the collector plates and the area of the limiting aperture. However, there is an error involved in considering only the radiation that passes through this aperture, as the thickness of the diaphragm is not small compared to its distance from the source of radiation, and thus some of the radiation will pass through the edge of the diaphragm and enter the chamber. To estimate the magnitude of this error it was necessary to determine what fraction of the total radiation producing ionization in the chamber was transmitted through the edge of the diaphragm. This was done by considering the activity of the source to be distributed uniformly over the surface of a thin disk located at the center of the source (fig. 8). If $\rho$ and $\theta$ are the polar coordinates of a point on the surface of the disk and $r$ is the distance from the source-diaphragm-chamber axis in a fixed direction at a distance $D$ along the axis from the source, where $D$ is equal to or greater than the distance $d$ between the source and the outer diaphragm surface, then the total amount of radiation received by the ionization chamber is given by

$$
2 \pi \int_{0}^{r_{\max }} \int_{0}^{\rho_{\max }} \int_{0}^{2 \pi} e^{-\mu \delta(r, \rho, \theta)} \rho r d r \rho d \theta
$$

where $\delta$ is the thickness of lead traversed by gamma rays emitted at $\rho, \theta$, which pass through the point $r$, and $\mu$ is the linear absorption coefficient of lead for the radiation in question. The maximum value of $\rho$ is equal to the radius of the source, and the maximum value of $r$ is taken to be that radius at which the radiation intensity is negligible for all values of $\rho$ and $\theta$. The absorber thickness $\delta$ is given by

$\delta=d-$

$\frac{D\left\{\rho(r \cos \theta-\rho)+\sqrt{\rho^{2}(\rho-r \cos \theta)^{2}+\left(a^{2}-\rho^{2}\right)\left(r^{2}+\rho^{2}-2 r \rho \cos \theta\right)}\right\}}{r^{2}+\rho^{2}-2 r \rho \cos \theta}$ 


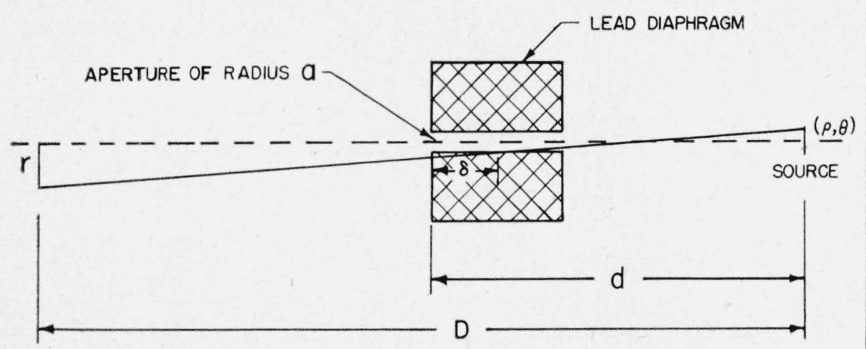

FIGURE 8. Schematic indication of the variables required for determination of the diaphragm leakage correction.

for all positive values of the right-hand term. When the right-hand term becomes negative, $\delta$ is equal to zero. This triple integral was evaluated by numerical integration, and the resulting intensity was compared with the intensity that was passed through the unobstructed aperture for the cesium and cobalt sources. In the case of cesium the correction was 2.40 percent, for cobalt it was 4.85 percent. These values are believed to be accurate to about 10 percent.

The resistance of the S. S. White resistor used in the grid circuit of the electrometer was measured during the course of the experiment and found to have a value of $3.003 \times 10^{9}$ ohms at $26^{\circ} \mathrm{C}$ and $3.161 \times 10^{9} \mathrm{ohms}$ at $23^{\circ} \mathrm{C}$. The precise temperature of the resistor during a set of measurements was not known but was taken to be the average value of the temperature of the air in the tank and room temperature. The maximum error due to this resistor is estimated to be about 1 percent.

By the application of the corrections due to air, aluminum, and Lucite absorptions [7], and that due to the diaphragm leakage, the correct dosage rates measured with the pressure and the extrapolation chambers were calculated. These values are tabulated in table 1, along with the thimble-chamber values. The cobalt source had been calibrated on June 1, 1949. The calibrated value corrected to the approximate date of this experiment was $5.05 \pm 0.15 \mathrm{r} / \mathrm{hr}$ at a distance equal to the source-diaphragm distance. The errors assigned to the various values have been estimated and consist largely of the errors already discussed.

TABLE 1. Comparative values of the dosage rates at the limiting diaphragm surface

\begin{tabular}{|c|c|c|}
\hline & Cesium-137 & Cobalt- 60 \\
\hline $\begin{array}{l}\text { Pressure chamber } \\
\text { Extrapolation chamber } \\
\text { Thimble chamber }\end{array}$ & $\begin{array}{c}r / h r \\
7.17 \pm 0.14 \\
7.03 \pm 0.21 \\
7.04 \pm 0.21\end{array}$ & $\begin{array}{c}r / h r \\
5.15 \pm 0.10 \\
5.17 \pm 0.15 \\
5.20 \pm 0.15\end{array}$ \\
\hline
\end{tabular}

Using the pressure chamber, the absorption coefficients for the gamma rays of cesium and cobalt were measured for lead, copper, and tin. Sets of standard absorbers ranging in thickness from 0.050 to about $3 \mathrm{~cm}$ were used in this determination. The values of the measured linear absorption coefficients are listed in table 2, along with the best available theoretical values [8]. The theoretical values are believed to be accurate within a few percent. The lower energy values are the most inaccurate because of the error in determining the more prominent photoelectric absorption coefficient.

TABLE 2. Linear absorption coefficients

\begin{tabular}{|l|r|r|r|r|r|r|}
\hline & \multicolumn{3}{|c|}{ Cesium-137 } & \multicolumn{3}{c|}{ Cobalt-60 } \\
\cline { 2 - 7 } & Lead & Tin & Copper & Lead & Tin & Copper \\
\cline { 2 - 7 } & & & & & \\
Measured & $c m^{-1}$ & $c m^{-1}$ & $c m^{-1}$ & $c m^{-1}$ & $c m^{-1}$ & $c m^{-1}$ \\
Calculated_.......... & 1.210 & 0.535 & 0.635 & 0.664 & 0.372 & 0.465 \\
.202 & .542 & .644 & .674 & .371 & .466 \\
\hline
\end{tabular}

\section{Conclusion}

The response of the pressure ionization chamber for radiation energies below $1.3 \mathrm{Mev}$ indicates that absolute dosage rates can be determined within a few percent in accordance with the international definition of the roentgen. The accuracy of the values obtained with this pressure chamber are limited mainly by three factors: (1) the error in the measurement of the ionization current, (2) the determination of the saturation current obtained by a JaffeZanstra extrapolation, and (3) the accuracy of the determination of the area of the diaphragm aperture, with the correction factor due to leakage of radiation through the edge of the diaphragm. A consideration of these façtors, together with the inherent tolerances of the chamber, led to an estimated error of 2 percent for the over-all accuracy of the results.

It appears that it is possible to get an accuracy that will allow dosage-rate measurements to be made with somewhat less than 1 percent error by minimizing the uncertainties arising from the sources listed above.

The dosage rates measured with the extrapolation and thimble chambers are in good agreement with the values determined with the pressure chamber. This is especially gratifying in the case of the thimble chamber, as it is used extensively and there has been some question regarding energy dependence for radiation in the million-electron-volt region. The Victoreen thimble chamber was not designed to measure the dose resulting from high energy radiation; however, it was found that the dose due to collimated cesium-137 and cobalt-60 gamma-rays could be determined relatively accurately by placing Lucite shells around the thimble to provide electronic equilibrium for the radiation in question.

\section{References}

[1] L. S. Taylor, G. Singer, and A. L. Charlton, Am. J. Roentgenol. Radium Therapy XLIV, No. 3 (1940).

[2] L. S. Taylor and G. Singer, Am. J. Roentgenol. Radium Therapy XLI, No. 2 (1939).

[3] L. M. Langer and R. D. Moffat, Phys. Rev. 78, 74 (1950).

[4] D. A. Lind, J. R. Brown, and J. W. M. DuMond, Phys. Rev. 76, 1838 (1949).

[5] H. Zanstra, Physica 2, 817 (1935)

[6] G. Failla, Radiology XXIX, 262 (1937).

[7] J. A. Victoreen, J. Applied Phys. 20, 1141 (1949).

[8] Gladys White, Tables of X-ray attenuation coefficients with introductory notes, NBS Report 1003 (May 7, 1951).

Washington, October 12, 1951. 